\title{
Complete transthoracic resection of giant posterior mediastinal goiter: case report and review of surgical strategies
}

This article was published in the following Dove Press journal:

OncoTargets and Therapy

22 April 2016

Number of times this article has been viewed

\author{
Honglin Zhao* \\ Dian Ren* \\ Yi Liu \\ Xin Li \\ YiWu \\ Gang Chen \\ Jun Chen
}

Department of Lung Cancer Surgery, Tianjin Lung Cancer Institute, Tianjin Medical University General Hospital, Tianjin, People's Republic of China

*These authors contributed equally to this work
Correspondence: Jun Chen; Gang Chen Department of Lung Cancer Surgery, Tianjin Lung Cancer Institute, Tianjin Medical University General Hospital, I54 Anshan Road, Heping District, Tianjin 300052, People's Republic of China Email huntercj2004@qq.com; chengang450@।63.com

\begin{abstract}
Intrathoracic goiters generally occupy anterior mediastinum, rarely involving the posterior mediastinal space. Reported herein is a 54-year-old female with a giant posterior mediastinal mass that was successfully resected via right posterolateral thoracotomy. The final pathologic diagnosis was giant posterior mediastinal goiter. This patient has done well postoperatively, with no evidence of local recurrence at 12-month follow-up. Related surgical strategies in past publications are summarized.
\end{abstract}

Keywords: intrathoracic goiter, posterior mediastinal mass, thoracotomy

\section{Background}

By definition, intrathoracic thyroid goiter (ITG) is largely $(>50 \%)$ confined to mediastinum ${ }^{1-4}$ thus accounting for $10 \%-15 \%$ of all mediastinal masses. However, anterior mediastinum is usually affected. Only $10 \%$ qualify as posterior mediastinal goiters (PMGs). ${ }^{5}$ Such growths typically are neuronal in origin, expanding as isolated mediastinal lesions or descending from the neck into substernal space. A variety of compressive symptoms may consequently result. Although goiters of anterior mediastinum are easily resected via transcervical route, PMGs may require extracervical access, such as posterolateral thoracotomy. ${ }^{6}$

\section{Patient presentation}

A 54-year-old female presented to our facility with progressive dyspnea and chest tightness. She denied hemoptysis or dysphagia. On physical examination, vital signs were normal, and no abnormalities of the neck were palpable. Laboratory diagnostics indicated normal thyroid function. Computed tomography (CT) revealed a giant cystic tumor of right posterior superior mediastinum adjoining the right lower lobe of thyroid gland. The mass was situated between superior vena cava and spine, shifting the compressed trachea to the left. Its lower margin extended past aortic arch (Figure 1A). Use of 99TcmO4tracer static imaging confirmed normal thyroid position, but tracer uptake varied within thyroid proper, and glandular contours were partly indistinct. In the lower right lobe, tracer appeared abnormally concentrated, whereas very sparse distribution was evident in the upper pole of right lobe and in the left lobe (Figure 1B). Bronchoscopy disclosed visible stenosis of the right airway owing to extrinsic pressure (Figure 1C).

In August of 2014, right posterolateral thoracotomy (at fourth intercostal space) was performed. The right posterior mediastinal tumor delineated preoperatively by CT was completely resected. Because no interconnection was evident, the right lobe of thyroid 


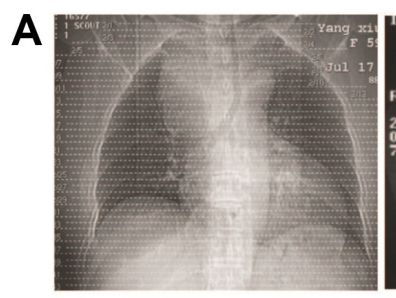

B

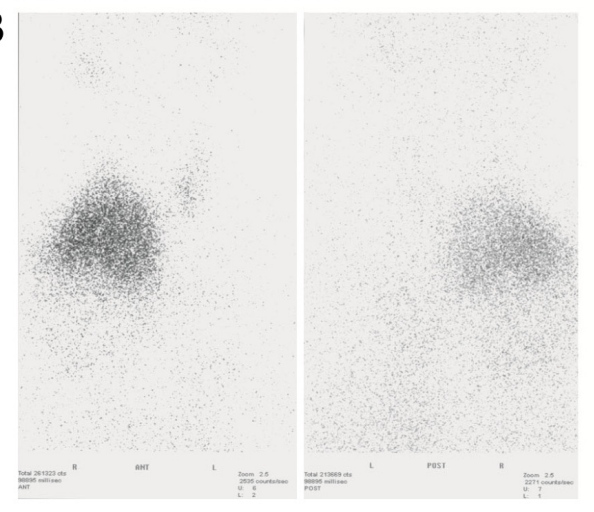

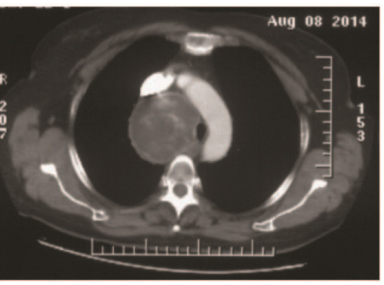

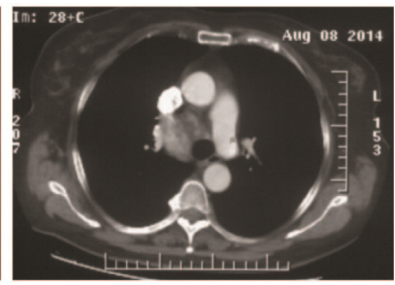

C
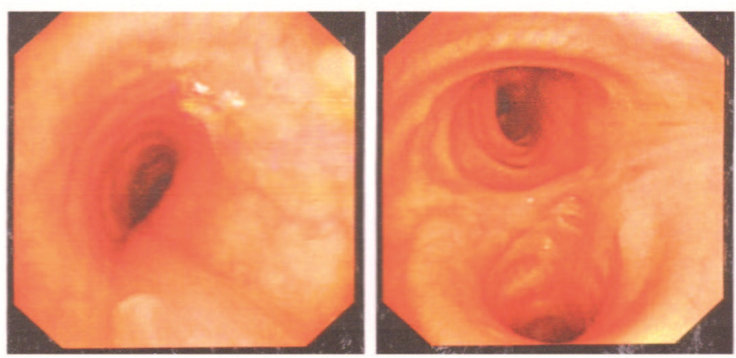

Figure I (A) Chest computed tomography scans of focally cystic, low-density posterior mediastinal mass (extending to carina) that compresses trachea and esophagus; (B) 99Tcm O4-tracer static imaging of normally positioned but indistinct thyroid gland; and (C) bronchoscopic images of visibly stenotic right airway suffering extrinsic mass effect.

was retained. The examining pathologist documented a large $(10.5 \times 7.2 \times 4.5 \mathrm{~cm})$, fully encapsulated, and hypervascular nodular goiter (Figure 2) with cystic and solid areas. The final diagnosis was giant goiter of posterior mediastinum. At 12 months postoperatively, the patient was symptom-free and had no signs of thyroid dysfunction.

Institutional Review Board of Tianjin Medical University General Hospital approved all procedures used in this case report. The patient granted written informed consent for publication of this report and accompanying images.

\section{Discussion}

ITGs constitute a small percentage of mediastinal tumors, most of which are benign. First described by Haller in 1749, these goiters are wholly or in part confined to mediastinum, expanding caudally at least $3 \mathrm{~cm}$ beyond sternal manubrium (with neck overextended). ${ }^{4}$ Such growths have either primary or secondary origin. Secondary ITGs generally arise from lower aspects of one or both lobes of a cervical thyroid and descend into mediastinal gaps. Primary ITGs are formed during embryonic development, at which time some or all thyroidal blastomeres are carried into the mediastinum as the heart and great vessels descend. ${ }^{7}$ Whereas secondary PMGs are usually supplied by inferior thyroid artery (via cervical thyroid gland), primary PMGs commonly are supplied by intrathoracic arterial branches, and their connection to cervical thyroid is marginal or absent. ${ }^{8}$ Most ITGs present in right mediastinum as thyroidal appendages. Expansion in

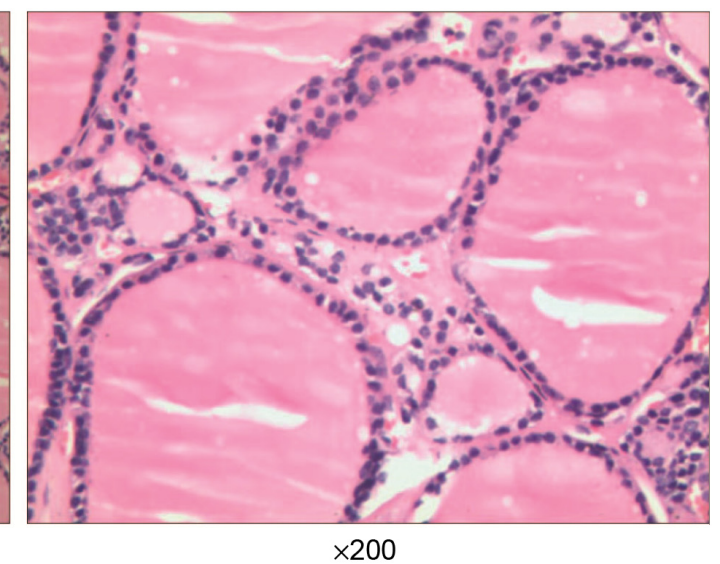

Figure 2 Microscopic views of large $(10.5 \times 7.2 \times 4.5 \mathrm{~cm})$ and fully encapsulated mass, diagnosed as hypervascular nodular goiter in ectopic thyroid tissue (hematoxylin and eosin stain). 
left mediastinum is apparently hampered by the thymus gland and the great vessels of mid-mediastinum, namely, superior vena cava or aortic arch and its three branches.

As cited by others, PMGs are asymptomatic in nearly $20 \%-30 \%$ of patients, discovered only as incidental findings on chest radiographs. ${ }^{9}$ As they enlarge, the most commonly reported manifestation $(40 \%-50 \%)$ is a cervical mass. Secondary symptoms due to intrathoracic airway compression include dyspnea, stridor, shortness of breath, and rhonchi. Less frequently, esophageal varices with digestive bleeding, superior vena cava syndrome, transient ischemic events, or cerebral edema may result from vascular compression. In $80 \%-90 \%$ of patients, a palpable cervical mass (among other signs) is observed. ${ }^{7,10-12}$

The most effective diagnostic modality for ITG is radiographic imaging. An upper mediastinal mass or related structural deformity, particularly tracheal deviation from midline and varying degrees of compression, is generally detectable by chest X-ray. CT provides precise information on spatial relationships of intrathoracic organs and is very useful in planning the surgical approach. Because iodine content dictates CT imaging density, ITG densities may vary (with respect to chest wall soft tissue) or appear uneven in tumors complicated by cysts or bleeding. ${ }^{13}$

Patients with ITG respond poorly to thyroxine treatment, although iodine therapy is an option in high-risk situations. Surgery is the treatment of choice in tumors that are unsuitable for medical management, may be malignant, or threaten acute airway compromise. ${ }^{14}$ Most secondary ITGs may be surgically removed via inferior cervical collar incision. Only $2 \%-3 \%$ of patients will require extracervical resection by an experienced surgeon, especially if a low-set primary ITG is involved (extending past aortic arch). ${ }^{15}$ However, both surgical approach and extent of surgery are subject to controversy. Partial resection is advised to protect recurrent nerves and parathyroid glands from surgical damage and because some tumors may recur 20-30 years after partial resection, total thyroidectomy is also suggested for patients with life expectancies $>10$ years. ${ }^{4}$ In this patient, an abundant blood supply from bronchial artery and aortic arch was confirmed intraoperatively, so despite some impingement on lower lobe of thyroid, the ITG was considered primary. Based on these findings and the functional status of cervical thyroid, the right lobe was left intact.

A number of surgical strategies are available for ITGs. The best approach is determined by several key factors, such as tumor size and location as well as the interrelationship with critical mediastinal structures. A collar incision is the usual choice because nearly all goiters $(>90 \%)$ with an intrathoracic component are accessible by this technique. ${ }^{12}$ Nevertheless, a cervicothoracic approach is required on occasion, especially in patients with primary ITGs or PMGs. Other resection techniques that are useful in this setting include partial or total median sternotomy, thoracotomy, and thoracoscopy. Median sternotomy is preferred and is indicated for the following: 1) very large ITGs supplied by intrathoracic vessels, 2) PMGs that displace or compress aortic arch or inflict superior vena cava syndrome, 3 ) recurrent goiters, and 4) malignant substernal goiters with lymph node metastasis. In posterior or mid-mediastinal ITGs that are closely associated with great vessels, a higher placed thoracotomy incision broadens the operative field, thus avoiding damage to surrounding vital tissues and enabling en bloc resection.

Given the many advantages of minimally invasive surgery, this option has become increasingly popular. Video-assisted thoracoscopic surgery reduces surgical risks, facilitates patient recovery, and has proven feasible for substernal goiter resection. Compared with thoracotomy, videoassisted thoracoscopic surgery is clearly beneficial (mortality, $0 \%$ vs $3.6 \%$; complications, $28 \%$ vs $45 \%$ ). The Da Vinci robot likewise has been utilized for ITG resection. In 2008, minimally invasive PMG resections were undertaken in two patients using the Da Vinci robot. However, this technique is quite difficult and is probably appropriate for very small goiters only. ${ }^{16}$ In our patient, the mass was too bulky for a minimally invasive approach, which at present is still in need of further validation.

Although rare, related surgical complications include postoperative tracheal collapse, prolonged mechanical ventilation, secondary hypocalcemia, damage to recurrent nerves, hematoma, hemorrhages, and incisional wound infections. A prospective study of 301 thyroidectomies performed for multinodular goiter found definitive complications in only $1 \%$. Furthermore, hyperthyroidism and goiter size were identified as risk factors for these complications. Currently, the primary routes of thyroid surgery are cervical incision, cervicothoracic access, thoracotomy, or thoracoscopic maneuvers. Each approach has advantages or disadvantages (Table 1). The proper approach depends on the patient's condition. Considering the size of our patient's tumor, its location, and its relationship with mediastinal structures, traditional thoracotomy was elected.

\section{Conclusion}

Lateral thoracotomy is advised for resection of giant PMGs, despite the inherent postoperative patient discomfort. 
Table I Surgical strategies for intrathoracic goiter

\section{Surgical approach}

Cervical incision: Most retrosternal goiters are resectable by cervical approach. The incision is limited and patient trauma is minimal, with brief postoperative hospitalization required. However, surgical risk is heightened due to inadequate visual exposure and reduced operative access. In giant ITGs, this approach is usually insufficient for complete resection and may increase chances of uncontrollable bleeding, damage to recurrent nerve, and other complications.

Thoracotomy: This route provides a wide operative field and is the best means of achieving complete tumor resection. Still, hospitalization time is prolonged, and related trauma often leads to complications, such as postoperative atelectasis and hypoxia. Recurrent, ectopic, invasive, malignant, or retrovascular goiters are generally subjected to thoracotomy.

Cervicothoracic access: This quick and reliable approach is excellent for easily controlling vascular injury and avoiding catastrophic results. The wide exposure facilitates complete tumor resection from both vantage points, but the trauma to patients is greater, exceeding that of cervical incision alone. This route is effective for acute upper airway obstruction and retrosternal goiter, especially PMGs extending past aortic arch.

Thoracoscopic techniques: Video-assisted thoracoscopy is a minimally invasive approach resulting in quicker recovery, less morbidity, less early postoperative pain, fewer days with chest tubes, shorter hospital stay, and faster return to work than open surgery. It also serves as a diagnostic technique and permits less usage of anesthetics. The drawbacks include an inability to control substantial hemorrhage and other emergencies. Overall, small goiters and primary ITGs are suitable indications for use. This complex technology requires professional training and presently is in need of further investigative validation.

Abbreviations: ITGs, intrathoracic thyroid goiters; PMGs, posterior mediastinal goiters.

The broad operative field afforded enhances surgical safety. In most patients treated surgically for ITG, the prognosis is good.

\section{Acknowledgments}

This research was partially supported by grants from the Science and Technology Support Key Program of Tianjin (12ZCDZSY16100) and the Tianjin Natural Science Foundation (12JCZDJC24400). Funding sources had no influence on study design, data collection, and analysis; on decision to publish; or on manuscript preparation.

\section{Author contributions}

$\mathrm{HZ}$ and $\mathrm{JC}$ generated this case report and analyzed all data. $\mathrm{GC}, \mathrm{HZ}$, and YL jointly performed the surgical procedure. DR,
YW, and XL rendered patient care and collected data. GC and JC together revised the article. All authors contributed toward data analysis, drafting and revising the paper and agree to be accountable for all aspects of the work.

\section{Disclosure}

The authors report no conflicts of interest in this work.

\section{References}

1. De Andrade MA. A review of 128 cases of posterior mediastinal goiter. World J Surg. 1977;1:789-797.

2. Chong CF, Cheah WK, Sin FL, Wong PS. Posterior mediastinal goiter. Asian Cardiovasc Thorac Ann. 2004;12:263-265.

3. Pieracci FM, Fahey TJ 3rd. Substernal thyroidectomy is associated with increased morbidity and mortality as compared with conventional cervical thyroidectomy. J Am Coll Surg. 2007;205:1-7.

4. Wu YH, Wein RO, Carter B. Thyroid hemiagenesis with multinodular goiter: a case presentation and review of literature. Laryngoscope. 2010;120 Suppl 4:S191

5. Katlic MR, Grillo HC, Wang CA. Substernal goiter. Analysis of 80 patients from Massachusetts General Hospital. Am J Surg. 1985;149: 283-287.

6. Kilic D, Findikcioglu A, Ekici Y, Alemdaroglu U, Hekimoglu K, Hatipoglu A. When is transthoracic approach indicated in retrosternal goiters? Ann Thorac Cardiovasc Surg. 2011;17:250-253.

7. Foroulis CN, Rammos KS, Sileli MN, Papakonstantinou C. Primary intrathoracic goiter: a rare and potentially serious entity. Thyroid. 2009; 19:213-218

8. Cichon S, Anielski R, Konturek A, Baczynski M, Cichon W, Orlicki P. Surgical management of mediastinal goiter: risk factors for sternotomy. Langenbecks Arch Surg. 2008;393:751-757.

9. de Aguiar-Quevedo K, Cerón-Navarro J, Jordá-Aragón C, et al. Bocio intratorácico. Revisión de la literatura médica [Intrathoracic goitre: A literatura review]. CIR ESP. 2010;88(3):142-145. Spanish.

10. Torre G, Borgonovo G, Amato A, et al. Surgical management of substernal goiter: analysis of 237 patients. Am Surg. 1995;61:826-831.

11. Rios A, Rodriguez JM, Canteras M, Galindo PJ, Tebar FJ, Parrilla P. Surgical management of multinodular goiter with compression symptoms. Arch Surg. 2005;140:49-53.

12. Rios A, Rodriguez JM, Galindo PJ, et al. Results of surgical treatment in multinodular goiter with an intrathoracic component. Surg Today. 2008;38:487-494.

13. Grainger J, Saravanappa N, D'Souza A, Wilcock D, Wilson PS. The surgical approach to retrosternal goiters: the role of computerized tomography. Otolaryngol Head Neck Surg. 2005;132:849-851.

14. Ket S, Ozbudak O, Ozdemir T, Dertsiz L. Acute respiratory failure and tracheal obstruction in patients with posterior giant mediastinal (intrathoracic) goiter. Interact Cardiovasc Thorac Surg. 2004;3: 174-175.

15. Ojanguren Arranz A, Baena Fustegueras JA, Ros Lopez S, et al. Best approach for posterior mediastinal goiter removal: transcervical incision and lateral thoracotomy. Arch Bronconeumol. 2014;50:255-257.

16. Cattaneo SM, Park BJ, Wilton AS, et al. Use of video-assisted thoracic surgery for lobectomy in the elderly results in fewer complications. Ann Thorac Surg. 2008;85:231-235; discussion 235-236. 
OncoTargets and Therapy

\section{Publish your work in this journal}

OncoTargets and Therapy is an international, peer-reviewed, open access journal focusing on the pathological basis of all cancers, potential targets for therapy and treatment protocols employed to improve the management of cancer patients. The journal also focuses on the impact of management programs and new therapeutic agents and protocols on

patient perspectives such as quality of life, adherence and satisfaction. The manuscript management system is completely online and includes a very quick and fair peer-review system, which is all easy to use. Visit http://www.dovepress.com/testimonials.php to read real quotes from published authors.

Submit your manuscript here: http://www.dovepress.com/oncotargets-and-therapy-journal 